Supplement of The Cryosphere, 14, 1519-1536, 2020

https://doi.org/10.5194/tc-14-1519-2020-supplement

(C) Author(s) 2020. This work is distributed under

the Creative Commons Attribution 4.0 License.

(c) (1)

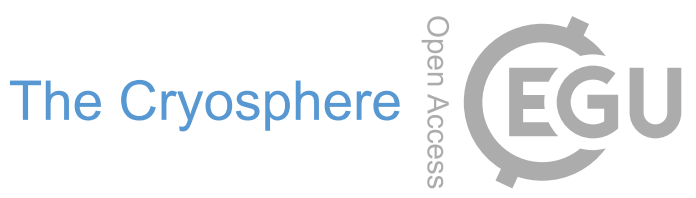

Supplement of

\title{
An enhancement to sea ice motion and age products at the National Snow and Ice Data Center (NSIDC)
}

Mark A. Tschudi et al.

Correspondence to: Mark A. Tschudi (mark.tschudi@ colorado.edu)

The copyright of individual parts of the supplement might differ from the CC BY 4.0 License. 


\section{S1. Evaluation of uncertainties in source data motion estimates}

The number and types of individual sources have changed over the ice motion product time series. Each source has different error characteristics based on the source and the method to estimate motion. As noted in the main text, Section 2.2, there have been several studies of motion estimate uncertainties, most focused on passive microwave motions derived from SSMI/SSMIS and AMSR-E imagery. These evaluations have been done via comparisons with buoy estimates. Buoy position is known very precisely and thus displacement and motion can be accurately retrieved. Thus, buoys act as a source of the "true" motion for validation.

Such comparisons were done early on in the product development and were used to derive the relative quality of the different source - i.e., the values of $C$ in Equation 1. These $C$ values have been used since without change. Here we provide a brief analysis of the source data via comparison with buoy source. This is not intended to be a complete validation study of each of the source motions. It is meant to give users of the product a general sense of the accuracy of the source motions.

The comparison method used all valid buoy motions over the given time period. For each buoy, the closest source motion estimate was found with a limit of $50 \mathrm{~km}$ (only source observations within $50 \mathrm{~km}$ of the buoy) were included. The $u$-component and $v$-component of motion, on the EASE grid, were compared between the source and the buoy for each day. All comparisons over the time period were used to calculate a bias (mean difference, e.g., $u$ source $-u$ buoy) and an Error Standard Deviation (Table S1). For each source, a typical year was chosen and statistics were calculated for winter (January through March) and summer (June through August). Particularly for the microwave estimates, this represents a period of optimal performance (winter) and a period with low performance (summer). For SMMR, summer estimates were very limited and there was not a large enough sample to effectively calculate statistics, so only winter is provided.

Overall, the statistics recapitulate what has been shown in previous studies. Passive microwave estimates generally have higher errors than AVHRR because of the lower spatial resolution. And spatial resolution of the source passive microwave imagery is a key factor in the error; higher spatial resolution imagery have lower Error Standard Deviation values. Note that SMMR Error 
33 Standard Deviation values are relatively low because SMMR imagery is every other day and the

34 daily value is a result of averaging between days, which reduces the noise in motion estimates.

One feature that is noticeable is the high errors in SSMI during summer, particularly the bias. This

37 is not surprising given the difficulties in retrieving surface properties during the melt season. This perhaps argues against inclusion of SSMI (and SSMIS, which has similar characteristics) in the combined product during summer. However, the approach used in the product is to use all available inputs throughout the year. During summer, the number of vectors is lower, particularly for 85

$41 \mathrm{GHz}$, so the impact of these lower quality vectors is relatively small compared to the buoys and

42 the complete field of wind-derived motions. AMSR-E is also affected by surface melt, but its

43 summer errors are not as high as SSMI, likely due to the higher spatial resolution.

Of note also is that the wind-derived motion error statistics are comparable to the passive microwave estimates, even lower in many cases (especially summer). This indicates that winds

47 should not necessarily be lower weighted than the other satellite sources. It also suggests that the 48 product's use of an ice/wind speed ration of $1 \%$ is not unreasonable, though $2 \%$ may yield 49 improvement, as shown in Table 4 of the main text.

51 This brief study indicates that the simplified values of $C$ are not optimal. This presents an avenue 52 to improve the combined motion product in a future version.

\section{S2. Validation of combined motions with CRREL buoys}

The combined daily motions are validated in the Arctic through comparison with independent

57 buoys from the CRREL Ice Mass Balance Buoy program [Perovich et al., 2020]. We compared

58 estimates to 101 CRREL buoys from 2000-2016. The total number of buoy-combined motion 59 observation pairs varied over 2000-2016, from a high of 3016 in 2007 to a low of 249 in 2002. A 60 small number $(<0.1 \%)$ of CRREL observations with erroneous velocities that were obviously too 61 large were removed from the comparisons. Overall, there are a total of 25,392 pairs over the 1762 year period, an average of 1494 per year. A map of the buoy tracks during 2015 is given in Figure 63 S1 as an example of the coverage and the trajectories of buoy motions. In 2015, the year used for 
64 the evaluation of the sensitivity of the wind-ice speed relationship, the 9 buoys resulted in a total 65 of 2025 paired observations.

67 All of the CRREL buoy positions were converted to EASE grid coordinates and the u-component 68 and v-component of velocity (relative to the EASE grid) was derived from the change in position over a 24-hour period. The combined gridded estimate closest to each CRREL buoy was selected for comparison; thus, each comparison was made generally within $\sim 25 \mathrm{~km}$. The results (Table S2)

71 show that biases are around $-0.1 \mathrm{~cm} / \mathrm{s}$ for the u-component and around -0.66 to $-0.69 \mathrm{~cm} / \mathrm{s}$ for the

72 v-component. Most notably, the biases were slightly reduced in Version 4, indicating that the 73 improvements in processing do result in improved accuracy of the motions. Similarly, the error 74 standard deviations are around $4 \mathrm{~cm} / \mathrm{s}$ for both velocity components and Version 4 reduces this 75 error by $\sim 0.3 \mathrm{~cm} / \mathrm{s}$ over Version 3.

A 2-dimensional histograms of the distributions of the errors relative to the CRREL buoys (Figure 78 S2), shows more detailed error characterization. The largest number of buoy-combined motion 79 pairs have differences $<1 \mathrm{~cm} / \mathrm{s}$. Version 4 shows slightly more pairs within this range, indicating an improvement over Version 3, in agreement with the statistics in Table S2.

As noted in Section 2.1 of the main text on Reanalysis winds, for the wind forcing, we used a $1 \%$ scale factor for the ice speed relative to wind speed. Other assessments have shown that $2 \%$ may 84 be more legitimate, especially in recent years with the observed positive trend in ice speed. To investigate the potential effect of underestimating ice speed from winds in our product, we compared the combined motion fields with both $1 \%$ and $2 \%$ scale factors to the 2015 CRREL buoy

87 observations. There were a total of 2025 comparison for 2015 . The results indicate little effect due to wind speed (Table S3), which is expected since the weighting of the wind-driven motion is

89 lower than other sources. The comparison indicates that the magnitude of the bias changes little 90 for the u-component, but actually increases for the v-component. The error standard deviations 91 decrease, generally by $\sim 0.5 \mathrm{~cm} / \mathrm{s}$. This suggests that $2 \%$ may indeed be an improvement, but the 92 impact on the combined gridded is relatively small. Of course, the relationship between wind speed 93 and ice motion is complicated and can be quite variable. It depends on the compactness of the ice 94 cover, thickness, and wind direction relative to nearby coasts. We plan to investigate the 
95 relationship further in the future, both regionally (for different sea ice conditions) and temporally

96 (to investigate the effect of the long-term trend toward increasing speeds).

97

98

99

100

101

102

103

104

105

106

107

108

Table S1. Error estimates for selected periods from the source motion estimates. All values are in $\mathrm{cm} / \mathrm{s}$. The Number column indicates the total number of buoy-source matches used in the statistics. The bias columns given the average difference in the motion components (source minus buoy) and the SD diff column provides the standard deviation of the difference.

\begin{tabular}{|c|c|c|c|c|c|c|c|}
\hline Year & Source & Season & Number & $u$ bias & $v$ bias & $u$ SD diff & $v \mathbf{S D}$ diff \\
\hline 1985 & SMMR & JFM & 284 & -0.18 & 0.06 & 3.66 & 3.39 \\
\hline \multirow[t]{2}{*}{1995} & \multirow[t]{2}{*}{ AVHRR } & JFM & 271 & 0.70 & 0.29 & 4.00 & 3.53 \\
\hline & & JJA & 80 & -0.53 & 0.29 & 4.95 & 3.78 \\
\hline \multirow[t]{8}{*}{2005} & \multirow[t]{2}{*}{ AMSR-E } & JFM & 1195 & -0.07 & -0.20 & 3.78 & 3.68 \\
\hline & & JJA & 175 & 0.67 & 1.02 & 4.53 & 4.98 \\
\hline & \multirow[t]{2}{*}{ SSMI 37} & JFM & 103 & 0.00 & 1.30 & 6.44 & 8.84 \\
\hline & & JJA & 93 & -2.52 & 0.96 & 7.64 & 10.32 \\
\hline & \multirow[t]{2}{*}{ SSMI 85} & JFM & 964 & -0.05 & 0.16 & 4.57 & 4.53 \\
\hline & & JJA & 69 & -2.07 & 3.67 & 6.79 & 9.14 \\
\hline & \multirow[t]{2}{*}{ Wind } & JFM & 1254 & 0.48 & 0.73 & 4.36 & 5.13 \\
\hline & & JJA & 2101 & 0.55 & 0.72 & 4.15 & 4.45 \\
\hline
\end{tabular}

Table S2. Validation statistics from comparison with CRREL buoys.

\begin{tabular}{|c|c|c|}
\hline & $u$-component $(\mathrm{cm} / \mathrm{s})$ & $v$-component $(\mathrm{cm} / \mathrm{s})$ \\
\hline \multicolumn{3}{|l|}{ Bias } \\
\hline Version 3 & -0.115 & -0.687 \\
\hline Version 4 & -0.111 & -0.660 \\
\hline \multicolumn{3}{|c|}{ Error St. Dev. } \\
\hline Version 3 & 4.20 & 4.32 \\
\hline Version 4 & 3.90 & 4.03 \\
\hline
\end{tabular}

Table S3. Comparison with CRREL buoys of combined motions using different wind-speed scaling.

\begin{tabular}{lcc}
\hline \multicolumn{1}{c}{$\boldsymbol{u}$-component $(\mathbf{c m} / \mathbf{s})$} & $\boldsymbol{v}$-component $(\mathbf{c m} / \mathbf{s})$ \\
\hline Bias & & \\
\hline $1 \%$ of wind speed & -0.267 & -0.190 \\
\hline $2 \%$ of wind speed & -0.263 & -0.220 \\
\hline Error St. Dev. & & \\
\hline 1\% of wind speed & 4.95 & 4.43 \\
\hline $2 \%$ of wind speed & 4.47 & 3.99 \\
\hline
\end{tabular}




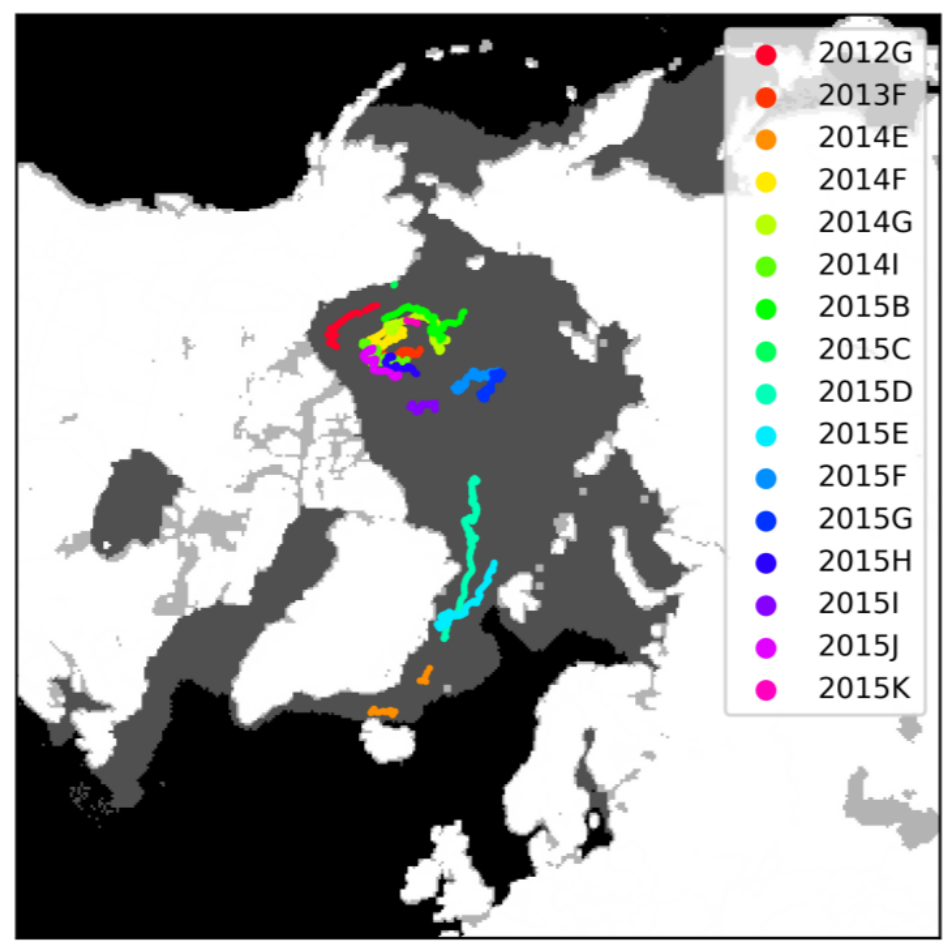

Figure S1. Map of CRREL buoy positions for 2015. The buoy IDs are color coded with the legend providing the CRREL buoy identifier; the year in the ID is the year of buoy deployment. All buoys that collected data during 2015 are shown except for buoys that were deployed on fast ice.

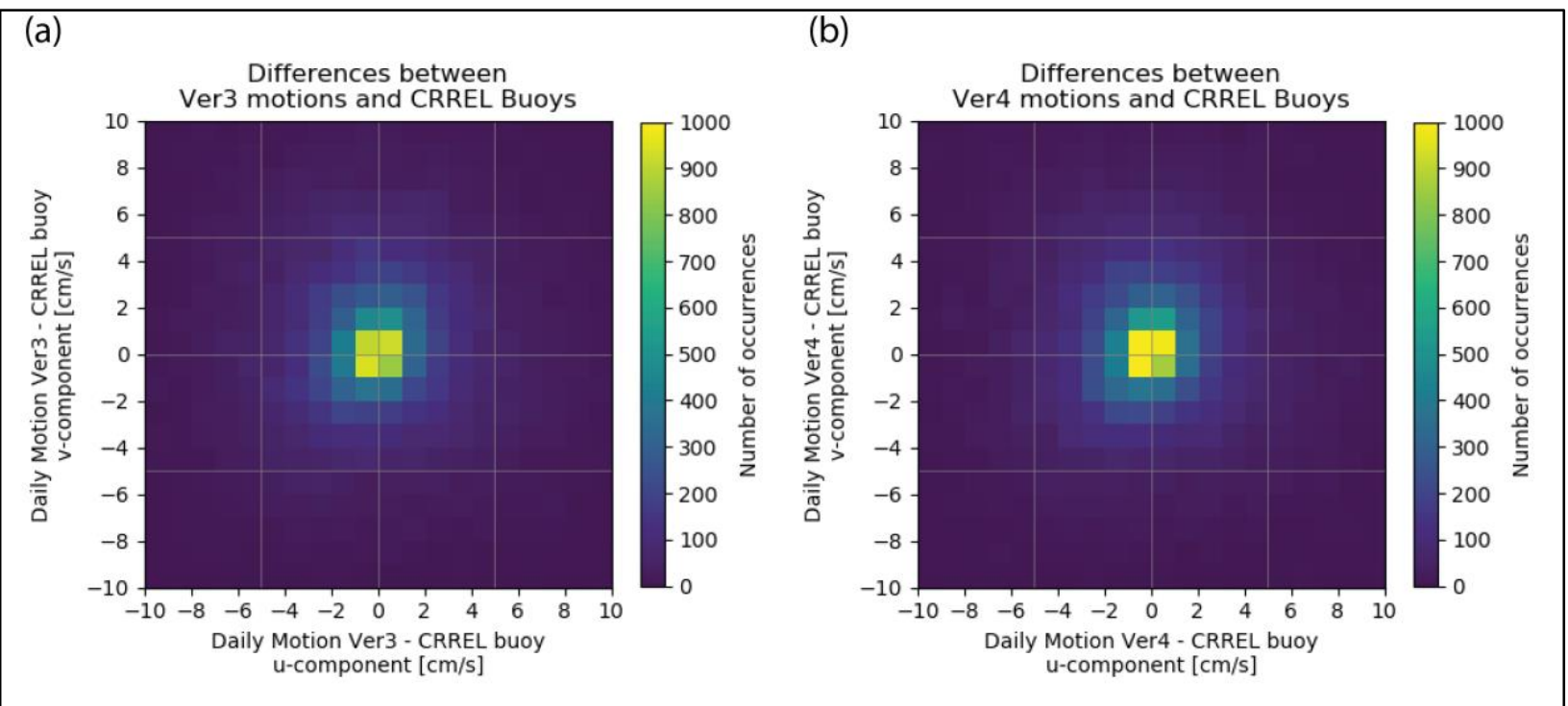

Figure S2. Two-dimensional histogram of combined motion error distribution relative to CRREL buoys for (a) Version 3 and (b) Version 4, binned into $1 \mathrm{~cm} / \mathrm{s}$ increments. The $v$-component error is on the $y$-axis with the $u$-component error on the $\mathrm{x}$-axis. The color scale indicates the number of occurrences within each bin. 
124 Supplemental References

125

126

127

128

129
Perovich, D., J. Richter-Menge, and C. Polashenski, Observing and understanding climate change: Monitoring the mass balance, motion, and thickness of Arctic sea ice, http://imb-crreldartmouth.org/archived-data, 2020. 